\title{
Reduced $\beta$-2-glycoprotein I inhibits hypoxia-induced retinal angiogenesis in neonatal mice through the vascular endothelial growth factor pathway
}

\author{
HONGYAN LIU ${ }^{1}$, SAIJUN ZHOU ${ }^{1}$, GARETH DENYER ${ }^{2}$, ZHENXING MENG ${ }^{1}$,

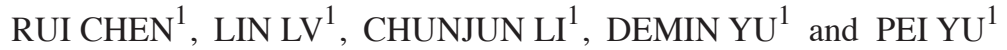 \\ ${ }^{1} 2011$ Collaborative Innovation Center of Tianjin for Medical Epigenetics, Key Laboratory of Hormones and Development \\ (Ministry of Health), Metabolic Diseases Hospital and Tianjin Institute of Endocrinology, \\ Tianjin Medical University, Tianjin 300070, P.R. China; ${ }^{2}$ Department of Biochemistry, \\ The University of Sydney, New South Wales 2006, Australia
}

Received October 17, 2013; Accepted July 23, 2014

DOI: $10.3892 / \mathrm{mmr} .2014 .2869$

\begin{abstract}
I ( $\beta 2 \mathrm{GPI})$, also known as apolipoprotein $\mathrm{H}$, is a phospholipid-binding plasma protein consisting of five homologous repeated units. $\beta 2$ GPI downregulates vascular endothelial growth factor (VEGF) signaling pathways and inhibits angiogenesis in vitro. However, the in vivo roles and effectors of reduced $\beta 2 \mathrm{GPI}$ and $\beta 2 \mathrm{GPI}$ in retinal angiogenesis are still not fully understood. In this study, an oxygen-induced retinopathy model was used to investigate the effects of reduced $\beta 2 \mathrm{GPI}$ and $\beta 2 \mathrm{GPI}$, and to monitor the expression of VEGF, VEGF receptor (VEGFR) 1, VEGFR-2 and hypoxia-inducible factor 1 (HIF-1) mRNA and the phosphorylation of extracellular signal-regulated kinase (ERK) and Akt. The data showed that both $\beta 2 \mathrm{GPI}$ and reduced $\beta 2 \mathrm{GPI}$ inhibited retinal angiogenesis and suppressed the expression of VEGF, VEGFR-1, VEGFR-2, HIF-1, phosphorylated- (p-) ERK and p-Akt. The effects of reduced $\beta 2$ GPI were significantly stronger than those of $\beta 2 \mathrm{GPI}$. In conclusion, this study showed that $\beta 2$ GPI and reduced $\beta 2$ GPI could inhibit retinal angiogenesis by downregulating the expression of VEGF and its downstream targets. This suggests that $\beta 2 \mathrm{GPI}$ and reduced $\beta 2$ GPI may have potential anti-angiogenic activity in vivo.
\end{abstract}

Correspondence to: Professor Pei Yu or Professor Demin Yu, 2011 Collaborative Innovation Center of Tianjin for Medical Epigenetics, Key Laboratory of Hormones and Development (Ministry of Health), Metabolic Diseases Hospital and Tianjin Institute of Endocrinology, Tianjin Medical University, 66 Tongan Street, Heping, Tianjin 300070, P.R. China

E-mail: yupei@tijmu.edu.cn

E-mail: yudemintij@126.com

Key words: reduced $\beta$-2-glycoprotein I, retinal angiogenesis, vascular endothelial growth factor

\section{Introduction}

Neovascularization is an important characteristic pathology of diabetic retinopathy (DR), retinopathy of prematurity and other hypoxic-ischemic retinal diseases (1). The resulting ectopic network of blood vessels can cause hemorrhage in the retina, choroid and vitreous, leading to retinal detachment and irreversible blindness. These diseases are one of the main causes of acquired blindness worldwide (2), and reliable animal models are required to further understand the underlying molecular mechanisms in order to develop preventative strategies.

The oxygen-induced retinopathy (OIR) model for research into retinal neovascularization was first described by Smith et al (3) in 1994. In this murine model there are two phases in the OIR pathological process: A $75 \%$ oxygen phase (hyperoxia) and a $21 \%$ oxygen phase (relative hypoxia). Hyperoxia induces the cessation of normal vessel growth and the regression of existing vessels, accompanied by a decrease in vascular endothelial growth factor (VEGF) expression. Relative hypoxia then induces an overexpression of pro-angiogenic substances, including VEGF, that causes the retina to initiate pathological neovascularization (3). Due to the pivotal role of VEGF in this angiogenesis, VEGF may be a potential novel therapeutic target for retinal neovascular diseases.

$\beta$-2-glycoprotein I ( $\beta 2 \mathrm{GPI})$, also known as apolipoprotein $\mathrm{H}$, downregulates VEGF signaling pathways and inhibits angiogenesis (4). Therefore, $\beta 2$ GPI represents a potential target in the treatment of DR. $\beta 2 \mathrm{GPI}$ is a phospholipid-binding plasma protein consisting of five homologous repeated units (domains I to V) (5). The cysteine (Cys) 288-Cys326 disulfide bond in domain $\mathrm{V}$ can be reduced by thiol oxidoreductase thioredoxin-1 or protein disulfide isomerase to form reduced $\beta 2$ GPI (6). Reduced $\beta 2$ GPI exhibits increased binding to von Willebrand factor through disulfide bonds and is able to protect endothelial cells, including EA.hy926 human endothelial cells, from oxidative stress-induced cell death in vitro $(7,8)$. Reduced $\beta 2$ GPI may therefore attenuate neovascularization through the 
amelioration of oxidative stress or through direct effects on VEGF activity.

In the present study, a mouse model of OIR was used to investigate the effects of $\beta 2 \mathrm{GPI}$ and reduced $\beta 2 \mathrm{GPI}$ on retinal neovascularization and to delineate the underlying molecular mechanisms.

\section{Materials and methods}

Animals and reagents. C57BL/6J newborn mice and nursing female mice were obtained from the Experimental Animal Center of Peking University Health Science (Beijing, China). $\beta 2$ GPI and reduced $\beta 2$ GPI were prepared as previously described (6). Evans Blue, diethylpyrocarbonate, EDTA and hematoxylin and eosin (H\&E) were purchased from Sigma-Aldrich (St Louis, MO, USA); RNasin Inhibitor and Moloney Murine Leukemia Virus (M-MLV) Reverse Transcriptase were purchased from Promega Corp. (Madison, WI, USA); SYBR ${ }^{\circledR}$ Premix Ex Taq ${ }^{\mathrm{TM}}$ DNA polymerase was purchased from Takara (Dalian, China) and TRIzol ${ }^{\mathrm{TM}}$ reagent was purchased from Invitrogen Life Technologies (Carlsbad, CA, USA). Rabbit anti-mouse polyclonal antibodies to Akt, phosphorylated- (p-) Akt, extracellular signal-regulated kinase 1/2 (ERK1/2), p-ERK1/2 and cluster of differentiation (CD) 34 were purchased from Bioworld Technology, Inc. (St. Louis Park, MN, USA). The quantitative polymerase chain reaction ( $\mathrm{qPCR}$ ) reaction primer sequences were designed by Primer 5.0 software (Premier Biosoft International, Palo Alto, CA, USA). and were synthesized by Beijing Genomics Institute (Beijing, China).

Animal model of proliferative retinopathy. All applicable institutional (Tianjin Medical University, Tianjin, China) and governmental regulations concerning the ethical use of animals were followed during this study. A reproducible model of OIR has been described previously in detail (3). Briefly, seven-day-old C57BL/6J mice, together with their nursing mothers, were exposed to $75 \pm 2 \%$ oxygen (hyperoxia) for five days before being returned to normal atmospheric conditions for a further five days. These mice were then randomly divided into three treatment groups and administered intravitreal injections of (respectively) $1 \mu 1$ phosphate-buffered saline (PBS), $1 \mu \mathrm{l} 800 \mu \mathrm{g} / \mathrm{ml} \beta 2 \mathrm{GPI}$ or $1 \mu \mathrm{l} 800 \mu \mathrm{g} / \mathrm{ml}$ reduced $\beta 2 \mathrm{GPI}$. Mice of the same strain and age were kept in normal atmospheric conditions for use as a control group.

Histological quantification of retinal neovascularization and immunohistochemistry. Mice were sacrificed by cervical vertebra dislocation. The eyes were enucleated, fixed with $4 \%(\mathrm{w} / \mathrm{v})$ paraformaldehyde in PBS and embedded in paraffin. Starting at the optic nerve head, a series of $6-\mu \mathrm{m}$, paraffin-embedded axial retinal sections were obtained, and these sections were stained with H\&E. Sections were examined for evidence of neovascularization by an individual blinded to the treatment groups using light microscopy. Neovascularization was quantified by the number of retinal vascular endothelial cell nuclei anterior to the inner limiting membrane (ILM). Counts were performed in 10 transverse sections through the center of the eye to produce an average number of neovascular cell nuclei/section/eye.
The paraffin sections underwent dewaxing, rehydration, antigen retrieval and treatment with $4 \%$ hydrogen peroxidase. Normal goat serum was used as a protein block and the sections were then incubated with rabbit anti-mouse polyclonal anti-CD34 antibody overnight at $4^{\circ} \mathrm{C}$. Following incubation with the primary antibody, the sections were incubated with biotinylated rabbit anti-mouse secondary antibody (diluted 1:200; Boster Biological Technology Co., Wuhan, China) for $20 \mathrm{~min}$ at $37^{\circ} \mathrm{C}$ and washed three times with PBS. Streptavidin-Horseradish Peroxidase Conjugate dilution buffer (Boster Biological Technology Co.) was subsequently applied for $20 \mathrm{~min}$ at $37^{\circ} \mathrm{C}$ prior to washing three times with PBS. The tissue sections were incubated in diaminobenzidine solution and counterstained with hematoxylin, prior to being dehydrated and mounted. PBS, in place of mouse monoclonal anti-CD34 antibody, was used as a negative control.

Angiography with Evans Blue. Mice were anesthetized by intraperitoneal injection of sodium pentobarbital $(300 \mathrm{mg} / \mathrm{kg})$ and were sacrificed by intracardiac perfusion with $1 \mathrm{ml}$ normal saline containing $30 \mathrm{mg} / \mathrm{ml}$ Evans Blue. The eyes were subsequently enucleated and fixed in $4 \%(\mathrm{w} / \mathrm{v})$ paraformaldehyde for $2-3 \mathrm{~h}$ at room temperature. The retinas were then dissected into four quadrants and flat-mounted onto microscope slides with neutral resin glue. Images, which were obtained by fluorescence microscopy, were analyzed using Adobe Photoshop (Adobe Systems Software Inc., San Jose, CA, USA) and the areas of non-perfusion and neovessels were calculated as a percentage of the total area of the retina.

Reverse transcription-qPCR. Enucleated eyes were used to prepare fresh retinas for RNA isolation. Total RNA was isolated using TRIzol ${ }^{\mathrm{TM}}$ reagent according to the manufacturer's instructions (Invitrogen Life Technologies). Having tested for nucleic acid integrity, an aliquot of RNA extract $(1.5 \mu \mathrm{g})$ was reverse-transcribed into cDNA using M-MLV Reverse Transcriptase. Primers were designed using Primer 5.0 software (Premier Biosoft, Palo Alto, CA, USA) and had the following sequences: VEGF forward, 5'-CTGGGCACT GCCTGGAAGAAT-3' and reverse, 5'-GGAAGATGAGGA AGGGTAAGC-3'; VEGFR-1 forward, 5'-CAAGCCAACGTC CAACAGGAT-3' and reverse, 5'-GCCCAGCAGAGTGCT AGTGTC-3'; VEGFR-2 forward, 5'-CAAGCCAACGTCCAA CAGGAT-3' and reverse, 5'-CCCTGAGTCAGCGTG AACTGC-3'; hypoxia-inducible factor-1 (HIF-1) forward, 5'-ATAAATGTTCTGCCCACCCTG-3' and reverse, 5'-GAC CCAACCACAAAGAGCAAG-3'; $\beta$-actin forward, 5'-TGG AGAAGAGCTATGAGCTGCCTG-3' and reverse, 5'-GTG CCACCAGACAGCACTGTGTTG-3'. The PCR was perfomed using an $\mathrm{iQ}^{\mathrm{TM}} 5$ Real-Time PCR Detection system (Bio-Rad Laboratories, Hercules, CA, USA). PCR cycling conditions were set as follows: $95^{\circ} \mathrm{C}$ for $5 \mathrm{~min}, 40$ cycles at $95^{\circ} \mathrm{C}$ for $30 \mathrm{sec}$, then $56^{\circ} \mathrm{C}$ for $30 \mathrm{sec}$ and $72^{\circ} \mathrm{C}$ for $30 \mathrm{sec}$. Melting curve analysis was subsequently performed between 55 and $95^{\circ} \mathrm{C}$ by monitoring fluorescence with $0.5^{\circ} \mathrm{C}$ increments at 30 -sec intervals. All sample measurements were performed in triplicate. Estimates of the quantity of the PCR product were obtained by densitometry using the Quantity One ${ }^{\circledR}$ analysis software package (Bio-Rad Laboratories). For each experimental 
A
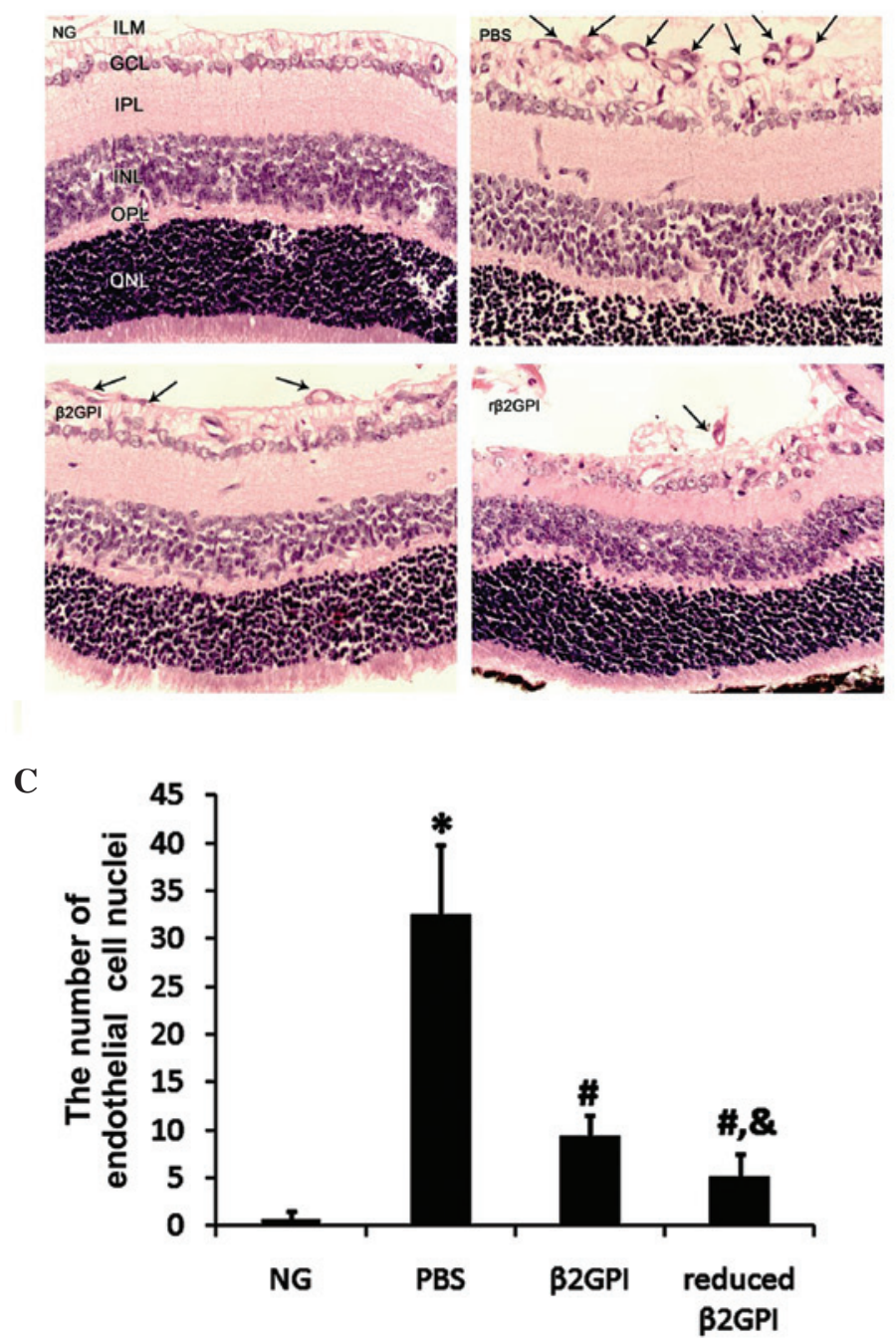

B
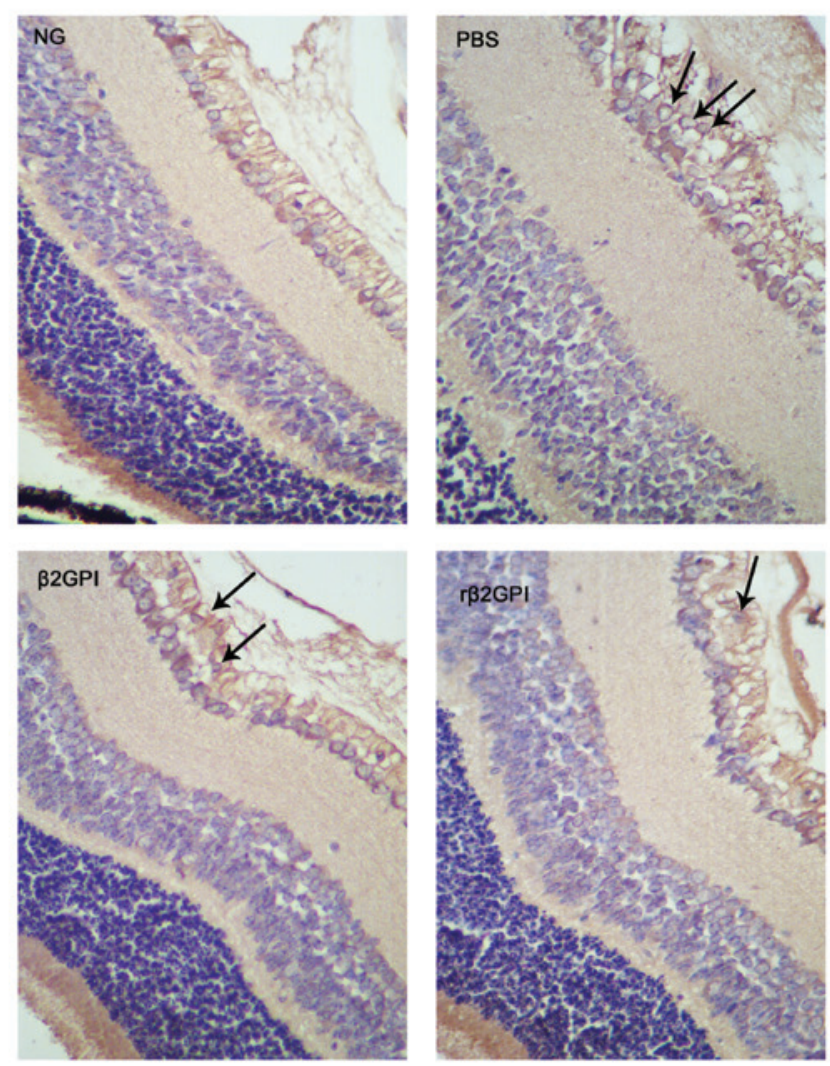

Figure 1. Histological quantification of retinal neovascularization. Neonatal mice were kept as controls or subjected to the oxygen-induced retinopathy protocol. (A) Neovascularization was assessed in hematoxylin and eosin-stained sections of the retina by counting the number of vascular endothelial cell nuclei (indicated by arrows) anterior to the ILM. (B) CD34 immunohistochemical staining of the retina was performed to reveal angiogenic endothelial cells (brown stain indicated by arrows). (C) Formal quantification of neovascularization observations. $\mathrm{n}=8$ in each group. Magnification, $\mathrm{x} 400$. Data are presented as the mean \pm standard error of the mean. ${ }^{*} \mathrm{P}<0.01$ vs. the NG group; ${ }^{\prime} \mathrm{P}<0.01$ vs. the PBS group; ${ }^{\&} \mathrm{P}<0.05$ vs. the $\beta 2 \mathrm{GPI}$ group. NG, neonatal controls; PBS, phosphate-buffered saline; $\beta 2$ GPI, $\beta$-2-glycoprotein I; r $\beta 2$ GPI, reduced $\beta 2 \mathrm{GPI}$; ILM, inner limiting membrane; GCL, ganglion cell layer; IPL, inner plexiform layer; INL, inner nuclear layer; OPL, outer plexiform layer; ONL, outer nuclear layer; CD, cluster of differentiation.

sample, target mRNA levels were normalized to $\beta$-actin mRNA levels.

Western blot analysis. Mouse retinas were collected and incubated in lysis buffer containing protease inhibitors (20 mM Tris, pH 7.4; $150 \mathrm{mM} \mathrm{NaCl} ; 1 \mathrm{mM}$ EDTA; $1 \mathrm{mM}$ phenylmethylsulfonyl fluoride; $1 \mathrm{mM}$ orthovanadate; $1 \mu \mathrm{g} / \mathrm{ml}$ leupeptin and $10 \mu \mathrm{g} / \mathrm{ml}$ aprotinin). Following homogenization and centrifugation, the amount of protein in the supernatant was determined with the bicinchoninic acid protein assay kit (Sigma-Aldrich). Aliquots of extract (35 $\mu \mathrm{g}$ protein per lane) from each sample were separated by SDS-PAGE using a $10 \%$ Tris-glycine gel prior to being transferred onto a polyvinylidene fluoride (PVDF) membrane at $65 \mathrm{~V}$ for $2 \mathrm{~h}$. Following blocking of the nonspecific binding sites by incubation with $5 \%$ skimmed milk for $1 \mathrm{~h}$, the membrane was incubated with rabbit anti-mouse polyclonal antibodies against Akt, p-Akt, ERK and p-ERK (1:1,000 dilution) overnight at $4^{\circ} \mathrm{C}$. The membrane was subsequently incubated with horseradish peroxidase-conjugated secondary antibody (goat anti-rabbit) for $1 \mathrm{~h}$ at room temperature. Peroxidase activity on the PVDF membranes was visualized on X-ray film with an ultra-violet transmission analyzer (GE Healthcare, Piscataway, NJ, USA).

Statistical analysis. Data were analyzed using SPSS 18.0 (SPSS Inc., Chicago, IL, USA). The results are expressed as the mean \pm standard error of the mean. One-way analysis of variance was used to evaluate statistically significant differences. $\mathrm{P}<0.05$ was considered to indicate a statistically significant difference.

\section{Results}

Histological quantification of retinal neovascularization and immunohistochemistry. The histological quantification and immunohistochemistry results are shown in Fig. 1. The OIR 

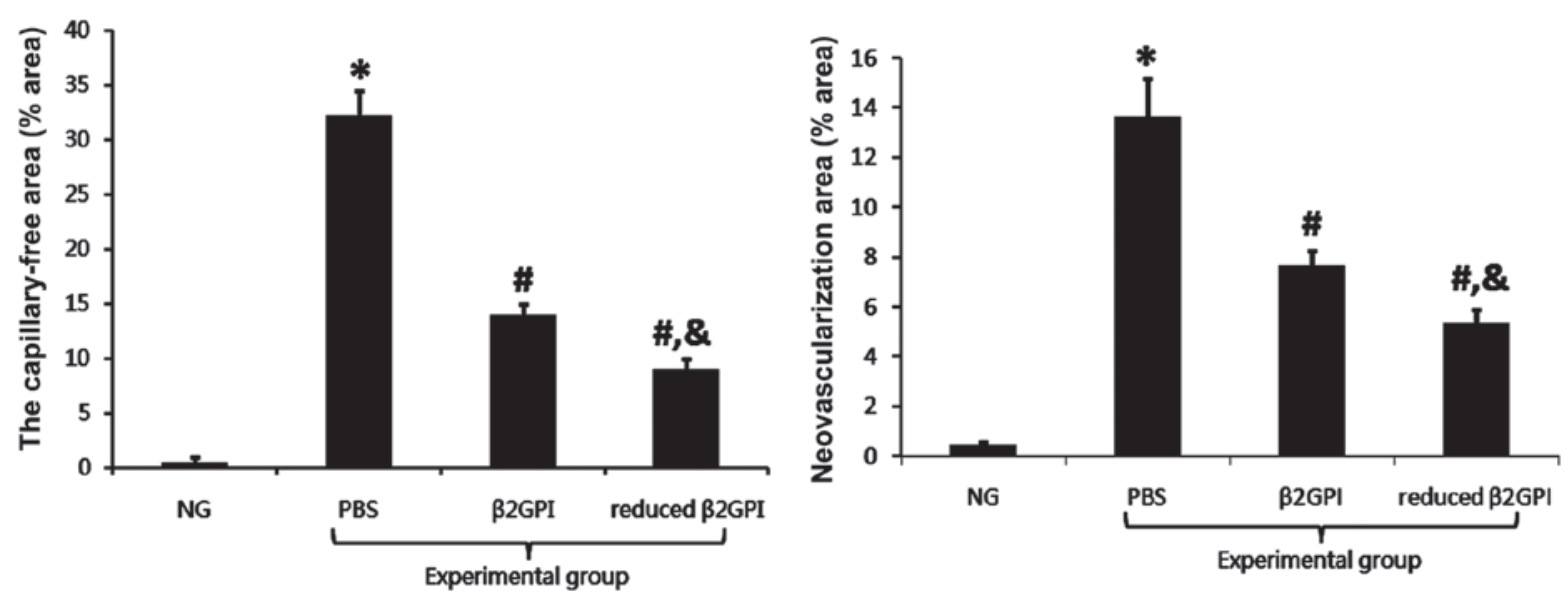

Figure 2. Formal quantification of the capillary-free and neovascularization areas. Prior to sacrifice, mice were administered an intracardiac perfusion containing $30 \mathrm{mg} / \mathrm{ml}$ Evans Blue. Enucleated eyes were fixed in 4\% paraformaldehyde, dissected into four quadrants, flat-mounted on microscope slides and observed with a fluorescence microscope. $\mathrm{n}=10$ in each group. Data are presented as the mean \pm standard error of the mean. ${ }^{*} \mathrm{P}<0.01$ vs. the normal group; ${ }^{\text {"}} \mathrm{P}<0.01$ vs. the PBS group; ${ }^{\circledR} \mathrm{P}<0.05$ vs. the $\beta 2 \mathrm{GPI}$ group. NG, neonatal controls; PBS, phosphate-buffered saline; $\beta 2 \mathrm{GPI}, \beta$ - 2 -glycoprotein I.

protocol resulted in a $>40$-fold increase in the number of endothelial cell nuclei anterior to the ILM (controls, $0.67 \pm 0.816$ vs. PBS, 32.5 $\pm 7.342 ; \mathrm{P}<0.05)$. Both $\beta 2 \mathrm{GPI}$ and reduced $\beta 2 \mathrm{GPI}$ significantly inhibited this increase $(9.33 \pm 2.16$ and $5.17 \pm 2.32$, respectively; $\mathrm{P}<0.01)$, with the effect of the reduced $\beta 2 \mathrm{GPI}$ observed to be statistically significantly stronger $(\mathrm{P}<0.05)$ (Fig. 1A and C). Immunohistochemical staining demonstrated that the cells anterior to the ILM were vascular endothelial cells (Fig. 1B).

Effects of $\beta 2$ GPI and reduced $\beta 2$ GPI on neovessels in the OIR mouse model. The vessels in the normal control group were mature and showed a normal radial distribution from the optic disc, as well as exhibiting a thicker diameter and a clear peripheral retinal structure, with the vessels forming vascular arcades along the peripheral retina. However, in the OIR PBS group, the diameter of blood vessels was reduced, and there was evidence of retinal neovessel formation, vessel leakage and an enlargement of the non-perfusion region. When formally quantified, the differences between the PBS and control groups were found to be statistically significant $(\mathrm{P}<0.01)$. Treatment with $\beta 2$ GPI resulted in restoration of vessel thickness. Furthermore, the formation of retinal neovessels, the vessel leakage and the enlarged non-perfusion region were significantly reduced as compared with the PBS group $(\mathrm{P}<0.01)$. This abrogation was even more marked with reduced $\beta 2$ GPI treatment (Fig. 2).

Effects of $\beta 2 G P I$ and reduced $\beta 2 G P I$ on $m R N A$ expression in retinopathy. The $\mathrm{mRNA}$ expression levels of VEGF, VEGFR-1, VEGFR-2 and HIF-1 were increased 2.5-4-fold by the induction of retinopathy $(\mathrm{P}<0.05$ or $\mathrm{P}<0.01)$ in the $\mathrm{PBS}$ group compared with the levels in the untreated control group. $\beta 2$ GPI and reduced $\beta 2$ GPI both inhibited this increase $(\mathrm{P}<0.05$ and $\mathrm{P}<0.01$ respectively), with the effect of reduced $\beta 2 \mathrm{GPI}$ observed to be stronger than that of $\beta 2 \mathrm{GPI}(\mathrm{P}<0.05$ or $\mathrm{P}<0.01)$ (Fig. 3).

Effects of $\beta 2$ GPI and reduced $\beta 2 G P I$ on the VEGF signaling pathway. Statistically significant increases in p-ERK1/2 and
p-Akt levels were observed in the PBS-treated group $(\mathrm{P}<0.05)$, as compared with the untreated control group. $\beta 2 \mathrm{GPI}$ inhibited this increase in $\mathrm{p}-\mathrm{ERK} 1 / 2$ in the retina $(\mathrm{P}<0.05)$; however, although the expression of p-Akt decreased following treatment with $\beta 2 \mathrm{GPI}$, this did not attain statistical significance. The expression of p-Akt and p-ERK1/2 in the retina showed a statistically significant decrease upon treatment with reduced $\beta 2$ GPI $(\mathrm{P}<0.05)$ when compared with the expression in the PBS and $\beta 2$ GPI groups (Fig. 4).

\section{Discussion}

Proliferative DR (PDR) is one of the most severe microvascular complications in patients with diabetes and is the major cause of acquired blindness. One important pathogenic mechanism in PDR is neovascularization through inappropriate angiogenesis. Passam et al (9) showed that the plasma glycoprotein $\beta 2$ GPI has the potential to reduce angiogenesis; through the same mechanism, $\beta 2$ GPI reduces the growth of tumor implants in mouse models (10). In the present study, it was shown that $\beta 2$ GPI exerts a stronger effect than $\beta 2$ GPI in its inhibition of neovascularization. These effects were regulated through VEGF and its important downstream targets, the phosphatidylinositol 3-kinase (PI3K)/Akt and mitogen-activated protein kinase (MAPK)/ERK signaling pathways. Angiogenesis is a complicated physiological process in vivo. In addition to angiogenic factors, such as VEGF, fibroblast growth factor and angiopoietin (11-14), this process includes anti-angiogenic factors: Platelet factor-4, the expression of which can be increased by $\beta 2$ GPI (15), angiostatin, thrombospondin-1 and numerous others (16-19). Angiogenic and anti-angiogenic factors maintain a dynamic balance under physiological conditions. A disruption of this balance can result in various pathologies. When anti-angiogenic factors dominate, the blood vessels are in a stationary or degradative state; when angiogenic factors dominate, the blood vessel formation process is initiated.

In this study, it was shown that reduced $\beta 2 \mathrm{GPI}$ inhibited retinal angiogenesis. This was accompanied by the downregu- 
HIF-1

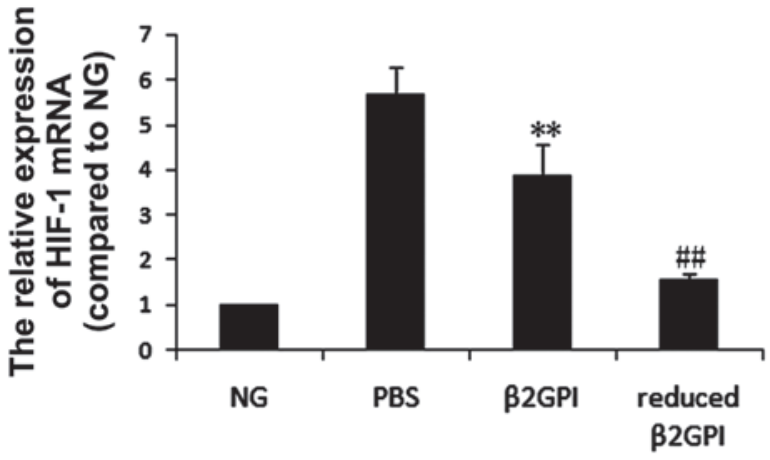

VEGFR-1

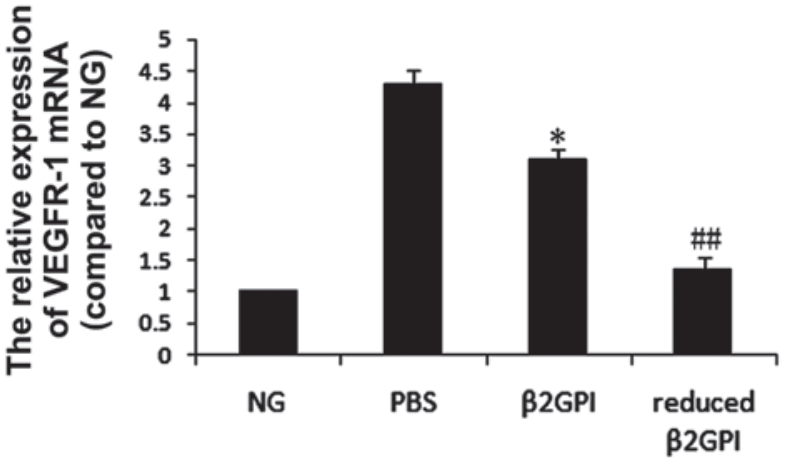

VEGF

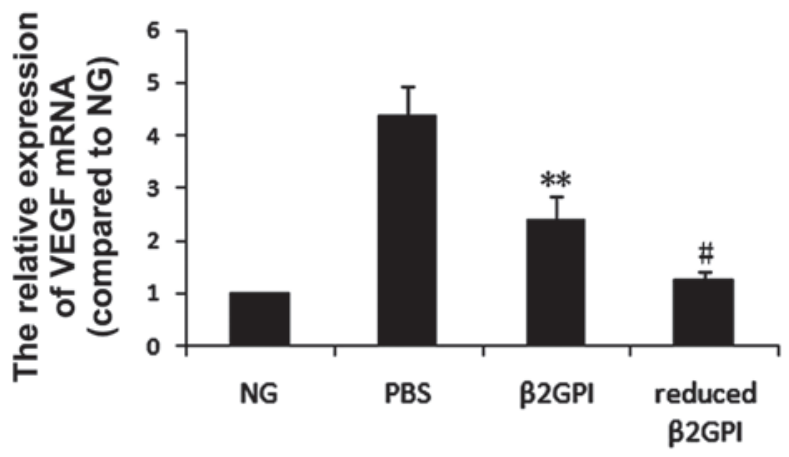

VEGFR-2

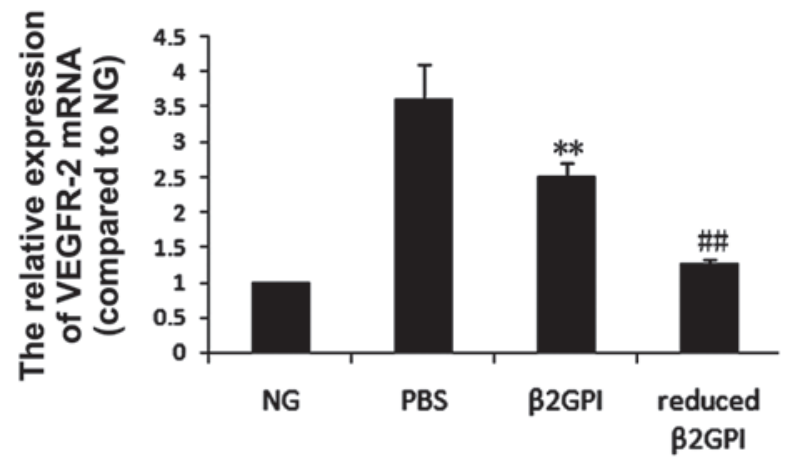

Figure 3. RT-qPCR analysis of mRNA expression in retinopathy. Following induction of retinopathy and treatment with PBS, $\beta 2$ GPI and reduced $\beta 2$ GPI, retinas were removed and the expression of selected mRNAs was measured using RT-qPCR. Estimates of the quantity of the PCR product were obtained by densitometry using the Quantity One ${ }^{\circledR}$ analysis software package. Results are presented as the mean \pm standard error of the mean of the mRNA level normalized to the $\beta$-actin mRNA level in the normal (non-retinopathy) group. $\mathrm{n}=15$ in each group. ${ }^{*} \mathrm{P}<0.05$ vs. the $\mathrm{PBS}$ group; ${ }^{* *} \mathrm{P}<0.01$ vs. the $\mathrm{PBS}$ group; ${ }^{\#} \mathrm{P}<0.01$ and ${ }^{\# \#} \mathrm{P}<0.01$ vs. the $\beta 2 \mathrm{GPI}$ group. PBS, phosphate-buffered saline; $\beta 2 \mathrm{GPI}, \beta$-2-glycoprotein I; RT-qPCR, reverse transcription-quantitative polymerase chain reaction; HIF-1, hypoxia-inducible factor 1; VEGF, vascular endothelial growth factor; VEGFR, VEGF receptor; NG, neonatal controls.

A
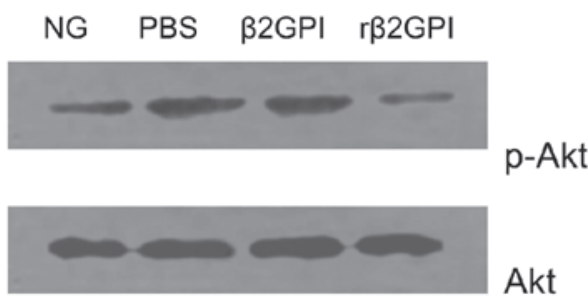

NG PBS $\quad \beta 2 G P I \quad r \beta 2 G P I$

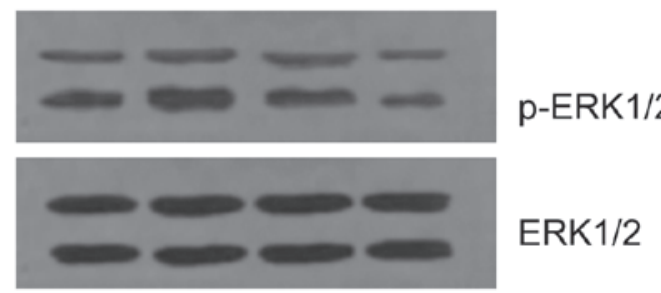

$\mathbf{B}$

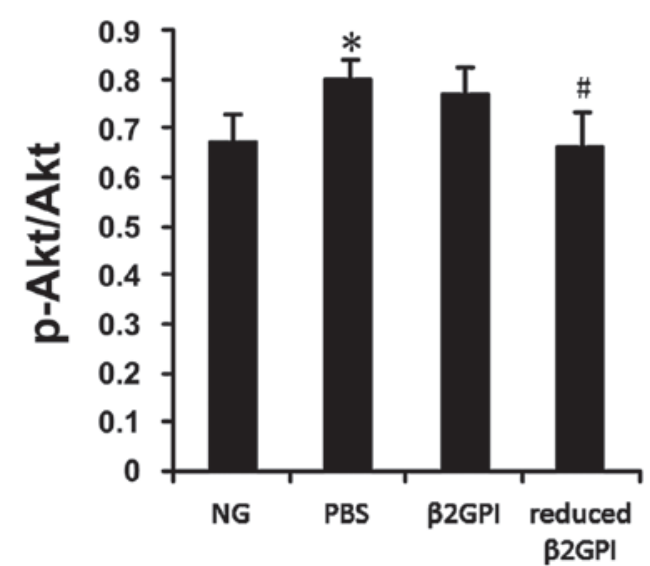

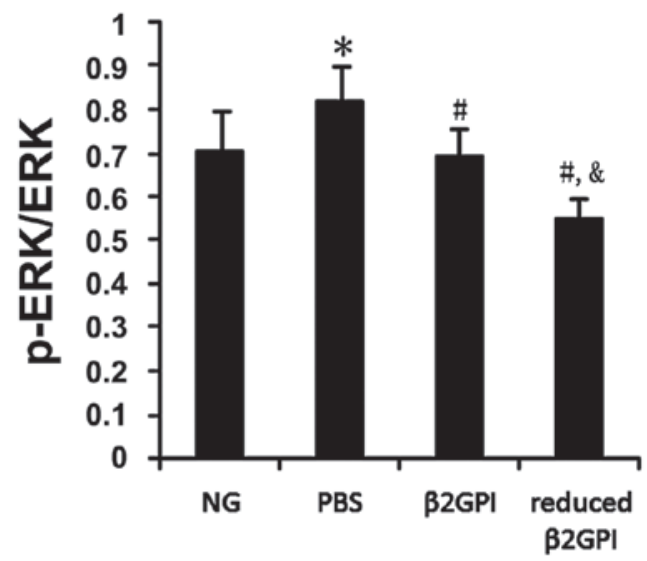

Figure 4. Effects of $\beta 2 \mathrm{GPI}$ and reduced $\beta 2 \mathrm{GPI}$ on Akt- and ERK-associated signaling in retinopathy. Extracts of retina from control (NG) and retinopathy-induced animals (PBS, $\beta 2 \mathrm{GPI}$ and reduced $\beta 2 \mathrm{GPI}$ ) were subjected to western blotting using the antibodies shown. (A) Representative images of blots probed with Akt, p-Akt (serine 473), ERK1/2 and p-ERK1/2 (threonine 202/tyrosine 204). (B) Average relative density of each band relative to that of $\beta$-actin from four separate experiments. "P $<0.05$ vs. the NG group; ${ }^{*} \mathrm{P}<0.05$ vs. the PBS group; ${ }^{\text {}} \mathrm{P}<0.05$ vs. the $\beta 2 \mathrm{GPI}$ group. NG, neonatal controls; PBS, phosphate-buffered saline; $\beta 2 \mathrm{GPI}, \beta$-2-glycoprotein I; p-, phosphorylated-; ERK, extracellular signal-regulated kinase. 
lation of VEGF, a key pro-angiogenic factor, and VEGFR-1/2. It remains unclear how reduced $\beta 2$ GPI downregulates VEGF and VEGFR; however, the data presented in this study showed that reduced $\beta 2 \mathrm{GPI}$ downregulates the expression of VEGF and VEGFR at the transcriptional level.

The PI3K/Akt signaling pathway influences angiogenesis and nutrient supply through the modulation of various factors, including endothelial nitric oxide synthase, HIF-1 and glycogen synthase kinase 3 (20,21), and promotes a survival response in endothelial cells, thus preventing apoptosis of these cells. This signaling pathway is also involved in preventing the degradation of newly-formed, immature lumen. Data from this study showed that reduced $\beta 2$ GPI significantly inhibited VEGF-induced Akt phosphorylation. ERK, a central protein of the MAPK/ERK signaling pathway (22), plays a key role in angiogenesis by entering the nucleus and activating nuclear factor $\kappa$-light-chain-enhancer of activated B cells $(23,24)$, followed by the induction of DNA synthesis and cell proliferation. The findings in the present study showed that reduced $\beta 2$ GPI blocked the phosphorylation of ERK1/2, thus suggesting this mechanism to be causative for the negative effect of reduced $\beta 2$ GPI on retinal angiogenesis.

In conclusion, $\beta 2 \mathrm{GPI}$ and reduced $\beta 2 \mathrm{GPI}$ may have potential anti-angiogenic activity in vivo. Both significantly inhibited pathological retinal angiogenesis, with the effect of reduced $\beta 2$ GPI observed to be stronger. $\beta 2 \mathrm{GPI}$ and reduced $\beta 2$ GPI were shown to act by downregulating the expression of HIF-1, VEGF and its receptors, VEGFR-1/VEGFR-2, on endothelial cells, and blocking the phosphorylation of ERK1/2 and Akt, downstream targets of VEGF in the MAPK/ERK and PI3K/Akt pathways. Intravitreal injection of reduced $\beta 2$ GPI to inhibit neovascularization may therefore provide a novel approach to the treatment of PDR.

\section{Acknowledgements}

The authors would like to thank the National Natural Science Foundation of China (nos. 30971393 and 81070645), the Tianjin Natural Science Fund (nos. 10JCYBJC12000 and 13ZCZDSYO1300), the Tianjin Health Bureau Natural Science Fund (nos. 09KZ01, 09KZ89 and 12KG135) and the Tianjin Medical University Science and Technology Fund (no. 2009ky25).

\section{References}

1. Tolentino MJ: Current molecular understanding and future treatment strategies for pathologic ocular neovascularization. Curr Mol Med 9: 973-981, 2009.

2. Wang FH, Liang YB, Zhang F, et al: Prevalence of diabetic retinopathy in rural China: the Handan Eye Study. Ophthalmology 116: 461-467, 2009.

3. Smith LE, Wesolowski E, McLellan A, et al: Oxygen-induced retinopathy in the mouse. Invest Ophthalmol Vis Sci 35: 101-111, 1994.

4. Yu P, Passam FH, Yu DM, Denyer G and Krilis SA Beta2-glycoprotein I inhibits vascular endothelial growth factor and basic fibroblast growth factor induced angiogenesis through its amino terminal domain. J Thromb Haemost 6: 1215-1223, 2008.
5. de Laat B, van Berkel M, Urbanus RT, et al: Immune responses against domain I of $\beta(2)$-glycoprotein I are driven by conformational changes: domain I of $\beta(2)$-glycoprotein I harbors a cryptic immunogenic epitope. Arthritis Rheum 63: 3960 -3968, 2011

6. Passam FH, Rahgozar S, Qi M, et al: Beta 2 glycoprotein I is a substrate of thiol oxidoreductases. Blood 116: 1995-1997, 2010.

7. Passam FH, Rahgozar S, Qi M, et al: Redox control of $\beta 2$-glycoprotein I-von Willebrand factor interaction by thioredoxin-1. J Thromb Haemost 8: 1754-1762, 2010.

8. Ioannou Y, Zhang JY, Passam FH, et al: Naturally occurring free thiols within beta 2-glycoprotein I in vivo: nitrosylation, redox modification by endothelial cells and regulation of oxidative stress induced cell injury. Blood 116: 1961-1970, 2010.

9. Passam FH, Qi JC, Tanaka K, Matthaei KI and Krilis SA: In vivo modulation of angiogenesis by beta 2 glycoprotein $\mathrm{I}$. J Autoimmun 35: 232-240, 2010.

10. Beecken WD, Engl T, Ringel EM, et al: An endogenous inhibitor of angiogenesis derived from a transitional cell carcinoma: clipped beta2-glycoprotein-I. Ann Surg Onco 13: 1241-1251, 2006.

11. Yoncopoulos GD, Davis S, Gale NW, Rudge JS, Wiegand SJ and Holash J: Vascular-specific growth factors and blood vessel formation. Nature 407: 242-248, 2000.

12. Yoshida S, Yoshida A and Ishibashi T: Induction of IL-8, MCP-1, and bFGF by TNF-alpha in retinal glial cells: implications for retinal neovascularization during post-ischemic inflammation. Graefes Arch Clin Exp Ophthalmol 242: 409-413, 2004.

13. Feng Y,vom Hagen F,Pfister F, et al: Impaired pericyte recruitment and abnormal retinal angiogenesis as a result of angiopoietin-2 overexpression. Thromb Haemost 97: 99-108, 2007.

14. Gardiner TA, Gibson DS, de Gooyer TE, de la Cruz VF, McDonald DM and Stitt AW: Inhibition of tumor necrosis factor-alpha improves physiological angiogenesis and reduces pathological neovascularization in ischemic retinopathy. Am J Pathol 166: 637-644, 2005.

15. Zhang R, Zhou SJ, Li CJ, et al: C-reactive protein/oxidised low-density lipoprotein/ $\beta 2$-glycoprotein I complex promotes atherosclerosis in diabetic BALB/c mice via p38mitogen-activated protein kinase signal pathway. Lipids Health Dis 12: 42, 2013.

16. Ljubimov AV, Caballero S, Aoki AM, Pinna LA, Grant MB and Castellon R: Involvement of protein kinase CK2 in angiogenesis and retinal neovascularization. Invest Ophthalmol Vis Sci 45: 4583-4591, 2004.

17. Uusitalo-Jarvinen $H$, Kurokawa $T$, Mueller BM, Andrade-Gordon P, Friedlander M and Ruf W: Role of protease activated receptor 1 and 2 signaling in hypoxia-induced angiogenesis. Arterioscler Thromb Vasc Biol 27: 1456-1462, 2007.

18. Qiao H, Sonoda KH, Ikeda Y, et al: Interleukin-18 regulates pathological intraocular neovascularization. J Leukoc Biol 81: 1012-1021, 2007.

19. Wu Z, Wang S, Sorenson CM and Sheibani N: Attenuation of retinal vascular development and neovascularization in transgenic mice over-expressing thrombospondin-l in the lens. Dev Dyn 235: 1908-1920, 2006.

20. Tchaikovski V, Olieslagers S, Böhmer FD and Waltenberger J: Diabetes mellitus activates signal transduction pathways resulting in vascular endothelial growth factor resistance of human monocytes. Circulation 120: 150-159, 2009.

21. Park JH, Lee JY, Shin DH, Jang KS, Kim HJ and Kong G: Loss of Mel-18 induces tumor angiogenesis through enhancing the activity and expression of HIF- $1 \alpha$ mediated by the PTEN/ PI3K/Akt pathway. Oncogene 30: 4578-4589, 2011.

22. Modi H, Li L, Chu S, Rossi J, Yee JK and Bhatia R: Inhibition of Grb2 expression demonstrates an important role in BCR-ABL-mediated MAPK activation and transformation of primary human hematopoietic cells. Leukemia 25: 305-312, 2011.

23. Parikh N, Shuck RL, Nguyen TA, Herron A and Donehower LA: Mouse tissues that undergo neoplastic progression after $\mathrm{K}$-Ras activation are distinguished by nuclear translocation of phospho-Erk1/2 and robust tumor suppressor responses. Mol Cancer Res 10: 845-855, 2012.

24. Taylor SM, Nevis KR, Park HL, et al: Angiogenic factor signaling regulates centrosome duplication in endothelial cells of developing blood vessels. Blood 116: 3108-3117, 2010. 